\title{
Synovial explant inflammatory mediator production corresponds to rheumatoid arthritis imaging hallmarks: a cross-sectional study
}

\author{
Martin Andersen 1,2, Mikael Boesen ${ }^{3}$, Karen Ellegaard', Robin Christensen', Kalle Söderström², Niels Søe , \\ Pieter Spee ${ }^{2}$, Ulrik GW Mørch ${ }^{5}$, Søren Torp-Pedersen ${ }^{1}$, Else Marie Bartels ${ }^{1}$, Bente Danneskiold-Samsøe , \\ Nina Vendel ${ }^{6}$, Lars Karlsson ${ }^{2}$ and Henning Bliddal ${ }^{1^{*}}$
}

\begin{abstract}
Introduction: Despite the widespread use of magnetic resonance imaging (MRI) and Doppler ultrasound for the detection of rheumatoid arthritis (RA) disease activity, little is known regarding the association of imaging-detected activity and synovial pathology. The purpose of this study was to compare site-specific release of inflammatory mediators and evaluate the corresponding anatomical sites by examining colour Doppler ultrasound (CDUS) and MRI scans.

Methods: RA patients were evaluated on the basis of CDUS and 3-T MRI scans and subsequently underwent synovectomy using a needle arthroscopic procedure of the hand joints. The synovial tissue specimens were incubated for 72 hours, and spontaneous release of monocyte chemoattractant protein 1 (MCP-1), interleukin 6 (IL-6), macrophage inflammatory protein $1 \beta(M I P-1 \beta)$ and IL-8 was measured by performing multiplex immunoassays. Bone marrow oedema (BME), synovitis and erosion scores were estimated on the basis of the rheumatoid arthritis magnetic resonance imaging score (RAMRIS). Mixed models were used for the statistical analyses. Parsimony was achieved by omitting covariates with $P>0.1$ from the statistical model.
\end{abstract}

Results: Tissue samples from 58 synovial sites were obtained from 25 patients. MCP-1 was associated with CDUS activity $(P=0.009$, approximate Spearman's $\rho=0.41)$, RAMRIS BME score $(P=0.01$, approximate Spearman's $\rho=0.42)$ and RAMRIS erosion score $(P=0.03$, approximate Spearman's $\rho=0.31)$. IL-6 was associated with RAMRIS synovitis score ( $P=0.04$, approximate Spearman's $\rho=0.50)$, BME score $(P=0.04$, approximate Spearman's $\rho=0.31)$ and RAMRIS erosion score ( $P=0.03$, approximate Spearman's $\rho=0.35)$. MIP-1 $\beta$ was associated with CDUS activity $(P=0.02$, approximate Spearman's $\rho=0.38)$ and RAMRIS synovitis scores $(P=0.02$, approximate Spearman's $\rho=0.63)$. IL-8 associations with imaging outcome measures did not reach statistical significance.

Conclusions: The association between imaging activity and synovial inflammatory mediators underscores the high sensitivity of CDUS and MRI in the evaluation of RA disease activity. The associations found in our present study have different implications for synovial mediator releases and corresponding imaging signs. For example, MCP-1 and IL-6 were associated with both general inflammation and bone destruction, in contrast to MIP-1 $\beta$, which was involved solely in general synovitis. The lack of association of IL-8 with synovitis was likely underestimated because of a large proportion of samples above assay detection limits among the patients with the highest synovitis scores.

\footnotetext{
* Correspondence: henning.bliddal@regionh.dk

${ }^{1}$ The Parker Institute, Department of Rheumatology, Copenhagen University Hospital, Bispebjerg and Frederiksberg, Nordre Fasanvej 57, Copenhagen 2000 Frederiksberg, Denmark

Full list of author information is available at the end of the article
}

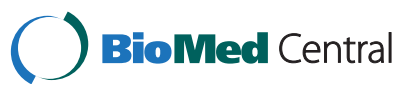

(c) 2014 Andersen et al.; licensee BioMed Central Ltd. This is an Open Access article distributed under the terms of the Creative Commons Attribution License (http://creativecommons.org/licenses/by/4.0), which permits unrestricted use, distribution, and reproduction in any medium, provided the original work is properly credited. The Creative Commons Public Domain Dedication waiver (http://creativecommons.org/publicdomain/zero/1.0/) applies to the data made available in this article, unless otherwise stated. 


\section{Introduction}

Rheumatoid arthritis (RA) is a chronic inflammatory disease characterised by progressive joint damage. The aetiology of RA is, to a large extent, unknown, and current therapies are aimed at reducing the inflammatory disease burden. Recent data support the practice of frequent monitoring of disease activity in RA patients to achieve remission faster [1,2]. However, patients may still experience radiographic progression of bone erosions, despite being judged to be in clinical remission [1,3,4]. The emergence of imaging techniques such as ultrasound (US) and magnetic resonance imaging (MRI) have paved the way for detailed descriptions of joint inflammation and erosion. These imaging modalities have recently been accepted as part of the diagnostic criteria in RA [1,5]. Because inflammatory activity detected by MRI or Doppler ultrasound (DUS) has shown to be a good predictor of disease course and response to treatment, the expansion of the use of these detection modalities in clinical assessments of RA has been suggested [6-8].

The invasive front of the synovium-the pannus-is believed to be the main driver of cartilage degradation and bone erosion; however, to the best of our knowledge, no researchers to date have investigated the association of histological features of synovitis with the extent of bone marrow oedema (BME) and erosions visualised by MRI. This information would be interesting because the presence of cluster of differentiation-positive (CD68+) macrophages in RA synovium has been linked to the development of erosions detected on radiographs [9]. Investigators who conducted MRI-based studies focused mainly on, and demonstrated, associations of MRI-detected synovitis and synovial histopathology [10-12] with MRI-detected BME and osseous histopathology [13-15]. Furthermore, previously published data have generally been based on small sample sizes. Also, the synovial material examined in the vast majority of cases was obtained from knee joints of patients undergoing knee joint replacement surgery in cases where inflammation due to concomitant osteoarthritis may have confounded the results. Current evidence is based mainly on studies in which the investigators assessed pathology on the basis of whole-joint imaging, which may have decreased sensitivity for the detection of local activity in the different areas of the synovium.

Studies of RA synovial explants have demonstrated the value of the capacity they provide for evaluating inflammatory output and the response to anti-inflammatory treatment, as well as for visualising the morphologic features of the RA synovium [16-24]. Several methods of establishing synovial cultures have been described, but the use of assays based on whole synovial tissue has been recommended in order to maintain synovial architecture and cell-to-cell contact [25]. Researchers in prior biopsy-based explant studies might not have taken the heterogeneous synovial tissue distribution into account, however, thus compromising the explant assays' ability to detect overall joint inflammation $[18,26]$. In recent years, novel immunoassay techniques, such as multiplex technology, have paved the way for simultaneous analysis of multiple cytokines and chemokines in a small sample. However, it has been shown that synovial production of heterophilic antibodies (HAs), such as rheumatoid factor (RF), can have a great impact on assay reliability if they are not blocked [27]. In contrast to synovial biopsies, explants consist of live cells, and the production of inflammatory mediators from these intact tissue cultures may thus provide novel information about RA pathogenesis.

Our aim in this study was to compare the association of synovial inflammatory mediator production with the colour Doppler ultrasound (CDUS) fraction [28] and/or the rheumatoid arthritis magnetic resonance imaging score (RAMRIS) [7] using 3-T MRI scans of the corresponding anatomical sites in the target hand. Obtaining synovial tissue by synovectomy under direct visual control enabled us to harvest as much of the synovium as possible from the different anatomical sites of the hand joints. The results of this study should thus provide novel insights into the association between synovial inflammatory mediators and RA hallmarks.

\section{Methods}

\section{Patients}

Patients with RA [29] and synovial hypertrophy diagnosed on the basis of greyscale US image analysis were eligible for the study. We excluded patients who were under 18 years of age, allergic to local anaesthetics, undergoing anticoagulant treatment or taking a prednisolone dose above $10 \mathrm{mg} /$ day, receiving intraarticular prednisolone injections into the hand, had synovectomy performed within the previous 3 months or had bad skin at the site of interest. Otherwise, no patient selection method was utilised. The enrolment period dated from June 2011 through January 2013. Patient recruitment and clinical and imaging examinations were performed at the outpatient clinic of the Department of Rheumatology at Copenhagen University Hospital, Bispebjerg and Frederiksberg, in Denmark. The study was approved by the local ethics committee of the capital region of Denmark (H-4-2009-117). The approval covered all centres involved in the study, and signed, informed consent was obtained from each patient. Further details about the centres involved are given in the Acknowledgements.

\section{Imaging modalities \\ Ultrasound examination}

The evaluation was performed by an experienced US specialist (KE or STP) [31], and CDUS was used to assess the vascularisation of the synovial tissue. The region 
of interest (ROI) was defined as the synovial tissue. The maximal systolic colour fraction $\left(\mathrm{CF}_{\max }\right)$ in the ROI was selected as a marker of synovial inflammation. Colour Doppler mode was chosen as a marker of synovial inflammation because the sensitivity of detecting synovial blood flow achieved with the LOGIQ E9 imaging system (GE Healthcare, Waukesha, WI, USA) was higher than that possible using the power Doppler modality [32]. US examinations were performed as previously described using a $15-\mathrm{MHz}$ center frequency linear array matrix [31]. In brief, the Doppler preset was adjusted for maximum sensitivity at low flow (pulse repetition frequency of $0.4 \mathrm{kHz}$, lowest wall filter of $45 \mathrm{~Hz}$ and $7.5-\mathrm{MHz}$ Doppler frequency), with Doppler gain just below noise level. This preset remained unchanged throughout the study period. In all patients, the wrists, proximal interphalangeal (PIP) and metacarpophalangeal (MCP) joints were examined by US to identify the joints with the most pronounced involvement. All examinations were performed from standardised dorsal and dorsolateral positions using specific anatomic landmarks in the US image in the various positions [33]. Following identification of the anatomic landmarks in the greyscale image, the Doppler was activated and, while keeping the landmarks in the image, the transducer was adjusted until the scan plane with the most Doppler activity was identified. The transducer was held in this position for a couple of heart cycles, whereupon the image was frozen. By using the cineloop function, the frames with maximum and minimum Doppler activity corresponding to the systole were stored and transferred to a processing program. The synovial Doppler activity was calculated as the ratio of the systolic CDUS pixel count per unit of greyscale pixel count, defined as $\mathrm{CF}_{\max }$ [28]. In the wrist, the synovial tissues in the radiocarpal (RC) and midcarpal $(\mathrm{MC})$ joints were evaluated separately if possible. Colour Doppler mode images were chosen to detect markers of synovial inflammation, because its sensitivity in detecting synovial blood flow on the LOGIQ E9 imaging system was higher than that of the power Doppler mode [32].

Ventral scanning positions were omitted because only the dorsal part of the joint was synovectomised. The examiner was blinded to all patient characteristics. A table describing the anatomical landmarks is provided in Additional file 1.

A table with definitions of the anatomical landmarks is provided in Additional file 1.

\section{Magnetic resonance imaging}

MRI scans were evaluated by an experienced radiologist (MB). All patients were examined while in a 3-T MRI scanner (MAGNETOM Verio; Siemens, Erlangen, Germany) using a 16-channel cardiac coil covering the target hand.
Patients were supine with their hands alongside the body as previously described [34]. Briefly, pre- and postcontrast-enhanced T1-weighted coronal and axial short tau inverted recovery (STIR) sequences were used to calculate synovitis, BME and erosion RAMRIS scores. The RAMRIS synovitis scores, which range from 0 to 3 , correspond to no, low, moderate and severe synovitis, respectively, based on subjective evaluation. We used the RAMRIS BME component to evaluate the extent of BME from 0 to 3, where each step corresponded to a $33 \%$ increase in BME. The RAMRIS erosion score ranged from 0 to 10 , with each step corresponding to $10 \%$ increments in bone area eroded in the anatomy of interest. The synovitis scores were performed at the $\mathrm{RC}$ and $\mathrm{MC}$ levels in the wrist. MRI-based scores for BME and erosion were averaged according to the anatomic location of the synovectomy or if synovectomy positions had been pooled. MB was blinded to all patient characteristics and US data.

\section{Procedures}

The needle arthroscopic procedures were carried out at Section of Hand Surgery, Department of Orthopedics, at Gentofte Hospital in Hellerup, Denmark. The synovial explant culture assay was conducted at the Section of Translational Immunology in the Biopharmaceutical Research Unit at Novo Nordisk, Måløv, Denmark. Needle arthroscopy was performed within 24 hours after clinical, US and MRI examinations. The joints (up to two per patient) that seemed most inflamed clinically and on CDUS were referred for synovectomy [30]. Portals were established laterally for access to the extensor tendons. The RC part of the wrist joint was found at the level of the scapholunate ligament. The radial, central and ulnar joints were located during synovectomies between the scaphoid bone and the distal radius, lunate bone and distal radius, as well as between the triquetral bone and the ulnar meniscus. The MC part of the wrist joint was detected by using a needle probe $1 \mathrm{~cm}$ distal from the transverse line of the RC part of the joint. Synovial tissue was obtained from three compartments (between the scaphoid and trapezoid, capitate and lunate, and triquetral and hamate bones, respectively). For the PIP and MCP joints, the landmarks for the portals were the small concavities in the dorsoulnar and dorsoradial directions for the central slip in the PIP joint and through the sagittal band in the MCP joint. With these portal locations, it was possible to obtain good joint visualisation and sufficient distance between the arthroscope, shaver and joint structures. To match imaging pathology with arthroscopic sampling of the synovium, the surgeon (NS) was not blinded to the patient's diagnosis, medication or US findings. The US description with localisation of Doppler activity and anatomic landmarks enabled the surgeon to sample synovium from the corresponding 
anatomical sites. MRI pathology was mapped to the synovial samples by use of the local RAMRIS score component corresponding to the synovectomised area.

\section{Synovial explant cultures}

The synovectomy product was transferred under sterile conditions into 15-ml tubes containing RPMI 1640 and GlutaMAX media (Gibco/Life Technologies, Grand Island, NY, USA), $10 \%$ foetal bovine serum, $2 \%$ heat-inactivated human serum, $1 \%$ penicillin and streptomycin (complete medium (CM)). The synovium-containing tubes were kept on wet ice until the explant culture was established and incubated less than 90 minutes postsurgery. The synovial tissue was centrifuged $\left(4^{\circ} \mathrm{C}\right)$ at $1,400 \mathrm{rpm}$ for 7 minutes. The $\mathrm{CM}$ supernatant was removed by suction, and synovial wet weight was determined. Synovial tissue was distributed in multiple wells at $2 \mathrm{mg} / 200 \mu \mathrm{l}$ of CM in a 96-well Nunc MicroWell plate with Nunclon Delta Surface (Thermo Fisher, Waltham, MA, USA) containing bovine bone slices (IDS DT1BON1000-96; Immunodiagnostic Systems Ltd. Boldon, UK). The synovial explant culture was placed in a sterile incubator at $37^{\circ} \mathrm{C}, 100 \%$ humidity and $5 \% \mathrm{CO}_{2}$ for 72 hours, after which supernatants were collected and stored at $<80^{\circ} \mathrm{C}$ until analysed.

Screening of synovial biomarkers of inflammation and testing for HA interference were performed on explant supernatants from eight study participants. The supernatants were assessed by utilising the Human InflammationMAP multiplex immunoassay (Myriad RBM, Austin, TX, USA), which consists of a combination of three multiplex panels to evaluate a total of 46 mediators. Because of budget limitations, one of the three multiplex panels was selected for the study. That panel consisted of 15 mediators, of which interleukin 6 (IL-6), IL-8, monocyte chemoattractant protein 1 (MCP-1) and macrophage inflammatory protein $1 \beta$ (MIP-1 $\beta$ ) could be measured reproducibly from the explant cultures. Subsequent experiments were focused on these analytes. A complete list of the mediators tested is given in Additional file 2. The significance of HA interference was tested prior to analysis by adding $200 \mu \mathrm{g} / \mathrm{ml}$ HeteroBlock (Omega Biologicals, Bozeman, MT, USA), which previously has been reported to block HA interference [27]. For further validation of abolishment of possible HA interference, supernatants were diluted in six serial dilutions from a twofold to sixty-four-fold dilution. Linearity was preserved for all cytokines upon dilution $\left(R^{2}=0.99\right.$; data not shown). Supernatant measurements were carried out at the laboratories of Myriad RBM using the Luminex multiplex platform (R\&D Systems, Minneapolis, MN, USA). Samples were transported in dry ice under continuous temperature measurements and access to dryice supplements. The average explant mediator production explant culture was used in the statistical analysis. Supernatants were added to $200 \mu \mathrm{g} / \mathrm{ml}$ HeteroBlock and run at a 100-fold dilution (stored at 2-fold dilution and further diluted 50-fold when analysed). The lowest limit of detection for the assay was defined by Myriad RBM as the lowest concentration of an analyte in a sample at which the coefficient of variation of replicate standard samples was $30 \%$. The lowest assay detection limits were $72 \mathrm{pg} / \mathrm{ml}$ IL-6, $26 \mathrm{pg} / \mathrm{ml}$ IL-8, $240 \mathrm{pg} / \mathrm{ml} \mathrm{MCP-1} \mathrm{and} 366 \mathrm{pg} / \mathrm{ml}$ MIP-1 $\beta$. Plate-to-plate variation was less than $10 \%$. MA had access to all patient data. However, none of the imaging data outcome measures were available at the time the explant cultures were established.

\section{Statistical analysis}

Data were clustered within patients; thus a linear mixed model was applied for the statistical tests in order to prevent double-counting errors with inflated standard errors. As previously described, parsimony in the statistical models was achieved by omitting design variables and covariates from the model if no statistical significance was determined $(P>0.1)$. For model optimisation purposes, square root and logarithmic transformations were applied to achieve an approximate Gaussian distribution of residuals. The Spearman's $\rho$ estimate was considered important to the overall visual data interpretation. Because of doublecounting, these Spearman estimates are referred to as approximate Spearman coefficients [35]. Statistical analyses were calculated using SAS version 9.2 software (SAS Institute, Cary, NC, USA). MA and RC had access to all data.

\section{Results}

\section{Patient and sample characteristics}

As Figure 1 shows, 25 patients were enrolled into the study, 12 of whom were recruited from a previous study [35]. Three patients who were offered participation declined, and an additional three patients were excluded on the basis of the US examination because of lack of synovial hypertrophy in the hand joints (Figure 1). From among the recruited patients, five (20\%) were referred for synovectomy and the remaining twenty (80\%) accepted our invitation to participate in the study. The patient population consisted primarily of RF and anticitrullinated peptide antibody (ACPA)-positive women with long-standing disease and mean Disease Activity Score in 28 joints/C-reactive protein (DAS-28-CRP) levels in the upper end of the moderate disease activity interval. The majority (52\%) of patients had high disease activity, and 36\% had moderate disease activity. Three patients had either low disease activity $(n=1)$ or were classified as in disease remission $(n=2)$ on the basis of DAS28 criteria. Nearly one-third $(n=8)$ were being treated with biological agents (abatacept $(n=1)$, etanercept $(n=2)$, infliximab $(n=4)$ and rituximab $(n=1))$. Most other patients were being treated with methotrexate as monotherapy or in combination with other 


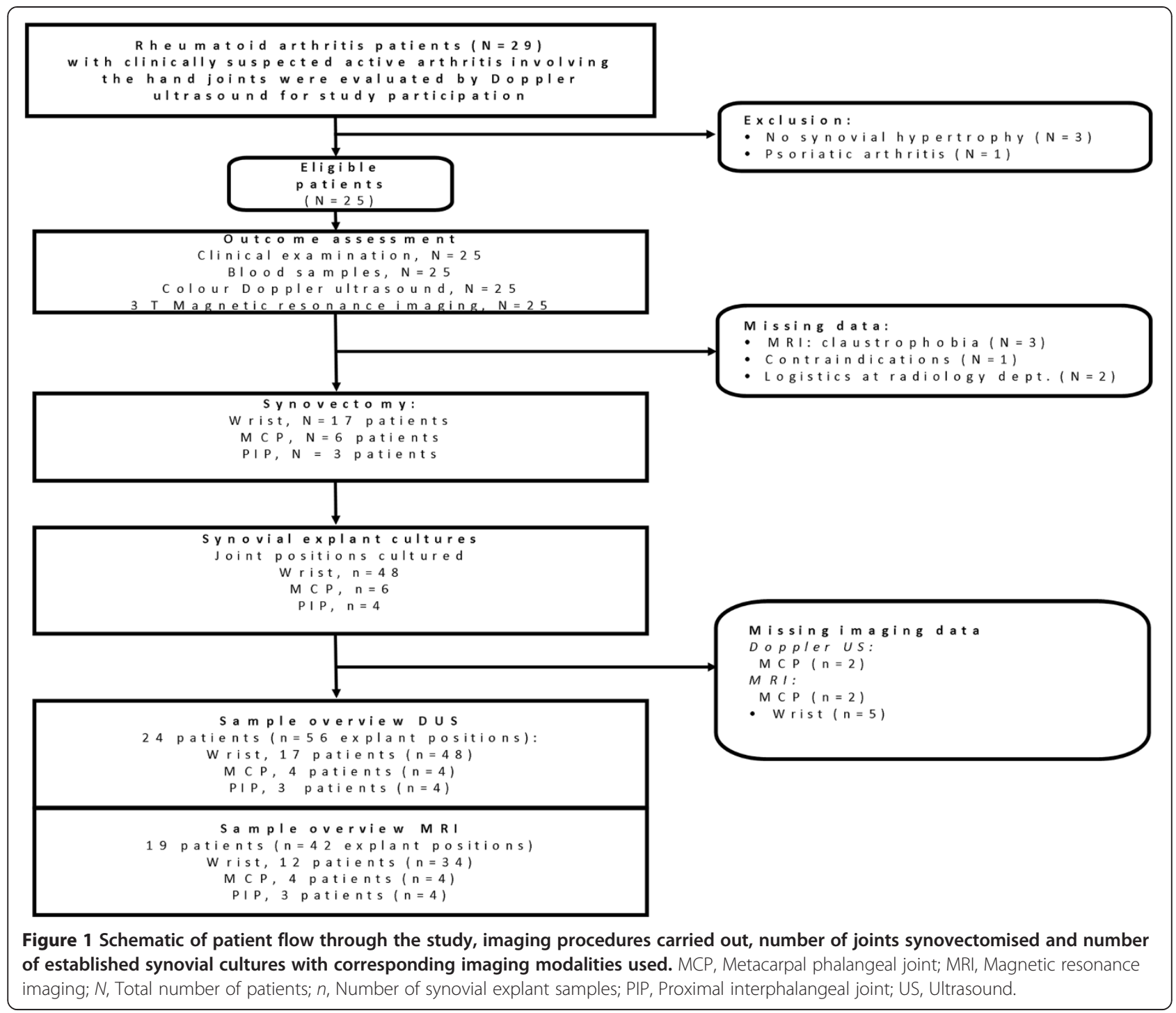

disease-modifying antirheumatic drugs (DMARDs) (28\% in each category) (Table 1).

\section{Synovial explant mediator production and imaging activity \\ Synovial explants}

A total of 71 synovectomy specimens were received from 26 joints (17 wrist joints, $6 \mathrm{MCP}$ joints and 3 PIP joints). From among the 71 synovectomies, 58 synovial explant cultures were established. On average, 22 wells were cultured per synovectomy position $(\mathrm{SD}=9.4)$. Three of the joint positions received were used for other purposes, and the remaining ten, due to sparse material, were pooled with one or more joint positions from the same joint. Synovectomy material was pooled from two cases, from two MCP joints in one case and from two PIP joints in the other case. In the cases where synovectomy material was pooled, an average of the imaging data from the corresponding positions was calculated. Three patients had two joints included (that is, two synovectomies), but only one patient with two joints synovectomised had corresponding imaging from both joints (MCP joints).

\section{Magnetic resonance imaging}

Nineteen (forty-two synovectomy positions) of twenty-five patients underwent MRI. As presented in Table 2, the mean RAMRIS BME component score was 2.05 (SD \pm 0.96 ), which corresponds to a moderate degree of osteitis ranging from one-third to two-thirds of the bone area. Three patients (seven synovectomies) had no BME. Synovial mediator levels among these patients were above detection limits for MCP-1 (6 (85\%) of 7 patients), IL-6 (4 (57\%) of 7 patients), IL-8 (7 (100\%) of 7 patients) and MIP-1 $\beta$ (1 (14\%) of 7 patients). Among the 16 patients (35 synovectomies) with $\mathrm{BME}>0$, levels of the four measured cytokines were above the assay's lowest detection limit in 35 
Table 1 Patient demographics and clinical characteristics ${ }^{a}$

\begin{tabular}{ll}
\hline Variables & Observations $(\boldsymbol{N}=\mathbf{2 5})$ \\
\hline Females, $n$ (\%) & $19(76 \%)$ \\
Mean age \pm SD, yr (IQR) & $56.2 \pm 14.9(26$ to 77$)$ \\
Disease duration, yr & $12.3[4.0$ to 14.1$](0.9 ; 42.7)$ \\
RF-positive, $n$ (\%) & $21(84 \%)$ \\
Anti-CCP-positive, $n$ (\%) & $22(88 \%)$ \\
Mean DAS-28-CRP score (0 to 10) & $4.8 \pm 1.3[2.2$ to 6.8] \\
CRP, mg/L & $20.9[4$ to 30] $(1 ; 117)$ \\
VAS global (scale 0 to 100) & $62.5[41$ to 83] \\
Tender joint count, 28 joints & $7.1[4$ to 9$](0 ; 25)$ \\
Swollen joint count, 28 joints & $7.8[4$ to 9] $(1 ; 21)$ \\
DMARD monotherapy & \\
MTX, $n$ (\%) & $2(8 \%)$ \\
SZS, $n$ (\%) & $3(12 \%)$ \\
LFU, $n$ (\%) & $2(8 \%)$ \\
DMARD combination treatment & \\
MTX + SZS, $n$ (\%) & $4(16 \%)$ \\
MTX + SZS + HCQ, $n$ (\%) & $3(12 \%)$ \\
Biologic DMARDs (\%) & $8(32 \%)$ \\
Prednisolone monotherapy, 5 mg/day & $2(8 \%)$ \\
No DMARDs (\%) & $1(4 \%)$ \\
\hline
\end{tabular}

${ }^{a}$ CCP, Cyclic citrullinated peptide; CRP, C-reactive protein; DAS28, Disease Activity Score in 28 joints; HCQ, Hydroxychloroquine; LFU, Leflunomide; MCP, Metacarpophalangeal joint; MTX, Methotrexate; PIP, Proximal interphalangeal joint; RF, Rheumatoid factor; SD, Standard deviation; SZS, Sulphasalazine; VAS, Visual Analogue Scale. Values are median [Q1 to Q3], (min; max) or mean \pm SD unless otherwise stated.

(100\%), 33 (94\%), 35 (100\%) and 16 (46\%), synovectomies, respectively.

\section{Ultrasound}

All 25 patients underwent CDUS. However, the synovectomised joint was not scanned in one patient because of a logistic accident; thus, 24 patients (56 synovectomy positions) were included for the statistical analysis. CDUS scans showed moderate activity on average, with a $\mathrm{CF}_{\max }$ of $12 \%$. However, 12 patients (48\%; 7 with matching CDUS and explant setup) had at least one US position with absence of Doppler activity. Among the 11 synovectomies (11 (20\%) of 55) cultured from these Doppler-negative positions, cytokine levels above the lowest detection limit were 9 (81\%) of 11 for MCP-1, 4 (64\%) of 11 for IL-6, 10 (91\%) of 11 for IL-8 and 5 (46\%) of 11 for MIP-1ß.

\section{Associations between imaging activity and synovial explant mediator production}

The statistical models show an association between levels of synovial IL-6 ( $P=0.04$, approximated Spearman's $\rho=$
$0.50)$ and MIP-1 $\beta(P=0.02$, approximated Spearman's $\rho=$ 0.63 ) and the RAMRIS synovitis score in both the reduced and full statistical models. IL-8 had statistically significant associations in all model reduction steps apart from the final step $(P=0.08$, approximated Spearman's $\rho=0.58)$, whereas MCP-1 did not have a statistically significant association $(P=0.17$, approximated Spearman's $\rho=0.48)$ with regard to the RAMRIS synovitis component score. Synovial perfusion, measured as $\mathrm{CF}_{\max }$, was associated with levels of MCP-1 ( $P=0.009$, approximated Spearman's $\rho=$ $0.41)$ and MIP-1 $\beta(P=0.02$, approximated Spearman's $\rho=$ 0.38). In contrast, IL-6 $(P=0.34$, approximated Spearman's $\rho=0.22)$ and IL-8 $(P=0.09$, approximated Spearman's $\rho=$ 0.27 ) were not statistically significantly associated with synovial perfusion. Figure 2 shows scatterplots for the statistically significant associations between RAMRIS synovitis score, $\mathrm{CF}_{\text {max }}$ and corresponding mediator release. Scatterplots illustrating imaging and explant mediator data not meeting the criteria for statistical significance are provided in Additional files 3 and 4.

Synovial MCP-1 $(P=0.01$, approximated Spearman's $\rho=0.42)$ and IL- 6 production $(P=0.04$, approximated Spearman's $\rho=0.25$ ) were associated with the RAMRIS BME component score and the RAMRIS erosion component score $(P=0.03$, approximated Spearman's $\rho=0.31$ for MCP-1) and $(P=0.03$, approximated Spearman's $\rho=0.35$ for IL-6).

Table 3 provides a general overview of the findings, including the remaining statistically insignificant findings. Figure 3 shows scatterplots of the data distributions regarding RAMRIS BME and erosion scores and synovial explant release. Additional files 5, 6, 7 and 8 provide details of the statistical model calculations, including the stepwise covariate elimination. All mediators that were statistically significantly associated with $\mathrm{CF}_{\text {max }}$, RAMRIS synovitis score and RAMRIS BME score were statistically significant in both the unadjusted and adjusted models. With regard to the RAMRIS erosion score, MCP-1 and IL-6 were above the statistical significance level in the unadjusted model $(P=0.07$ and $P=0.08$, respectively).

Table 3 presents the statistical associations between RA explant mediator release after 72 hours of culture and imaging activity. Imaging consisted of CDUS activity, reflected as the $\mathrm{CF}_{\max }$ in the systole. MRI-based activity outcomes were the RAMRIS components. A mixed model was used for the statistical analysis $(P<$ 0.05 was considered significant). In the reduced model, covariates were excluded when the $P$-value was $>0.10$. Table 3 contains the data from the reduced models. The full models, including stepwise covariate elimination steps and model optimisation transformation, are provided in the Additional files. The prespecified covariates included in the statistical model were joints 
Table 2 Overview of imaging observations and explant activity ${ }^{a}$

\begin{tabular}{|c|c|c|}
\hline Variable & $\begin{array}{l}\text { Observations No. patients } \\
\text { (No. synovectomies) }\end{array}$ & Median [IQR] (min;max) \\
\hline \multicolumn{3}{|l|}{ Imaging } \\
\hline \multicolumn{3}{|c|}{ Focal RAMRIS BME score, 0-3 } \\
\hline Wrist & $12(34)$ & $1.8[0.8$ to 3.0$](0.0 ; 3.0)$ \\
\hline MCP & $4(4)$ & $2.0[1.3$ to 2.8$](0.0 ; 3.0)$ \\
\hline PIP & $3(4)$ & $2.0[1.0$ to 2.5$](1.0 ; 2.5)$ \\
\hline Total & $19(42)$ & $1.5[1.0$ to 2.8$](0.0 ; 3.0)$ \\
\hline \multicolumn{3}{|l|}{$\mathrm{CF}_{\max }, 0-1$} \\
\hline Wrist & $17(48)$ & $0.08[0.02$ to 0.23$](0.0 ; 0.86)$ \\
\hline MCP & $4(4)$ & $0.12[0.05$ to 0.34$](0.03 ; 0.52)$ \\
\hline PIP & $3(4)$ & $0.06[0.03$ to 0.07$](0.0: 0.08)$ \\
\hline Total & $24(56)$ & $0.08[0.01$ to 0.21$](0.0 ; 0.86)$ \\
\hline \multicolumn{3}{|c|}{ Explant mediator production, $\mathrm{pg} / \mathrm{mL}$} \\
\hline \multicolumn{3}{|l|}{ MCP-1 } \\
\hline Wrist & $17(48)$ & $4,848[1,138$ to 15,000$](240.5 ; 46,450)$ \\
\hline MCP & $6(6)$ & $2,444[840$ to 11,175$](240.5 ; 31,336)$ \\
\hline PIP & $3(4)$ & $2,082[986$ to 2,707$](348 ; 3,053)$ \\
\hline Total & $25(58)$ & $3,865[1,138$ to 12,915$](240.5 ; 46,450)$ \\
\hline \multicolumn{3}{|l|}{ IL-6 } \\
\hline Wrist & $17(48)$ & $8,465[1,321$ to 39469$](72.5 ; 266,857)$ \\
\hline MCP & $6(6)$ & $4,969[391$ to 37,496$](72.5 ; 534,340)$ \\
\hline PIP & $3(4)$ & $11,901[1,761$ to 29,205$](732 ; 37,400)$ \\
\hline Total & $25(58)$ & $8,031[1,156$ to 37,400$](72.5 ; 534,340)$ \\
\hline \multicolumn{3}{|l|}{ IL-8 } \\
\hline Wrist & $17(48)$ & $21,129[5,649$ to 96,401$](26,5 ; 137,001)$ \\
\hline MCP & $6(6)$ & $21,132[5,405$ to 48,883$](4,571 ; 137,001)$ \\
\hline PIP & $3(4)$ & $20,730[6,388$ to 50,750$](2,017 ; 70,800)$ \\
\hline Total & $25(58)$ & $21,129[5,503$ to 89,643$](26.5 ; 137,001)$ \\
\hline \multicolumn{3}{|l|}{ MIP-1 $1 \beta$} \\
\hline Wrist & $17(48)$ & $1,812[530$ to 6,828$](366.5 ; 28,420)$ \\
\hline MCP & $6(6)$ & $1,078[577$ to 2,786$](366.5 ; 19,120)$ \\
\hline PIP & $3(4)$ & $555[354$ to 1,724$](366.5 ; 2,705)$ \\
\hline Total & $25(58)$ & $1,331[502$ to 5,196$](366.5 ; 28,420)$ \\
\hline
\end{tabular}

Depicting an overview of the imaging activity and synovial explant mediator production. Avr. = average, $\mathrm{CF}_{\max }=$ colour fraction measured in the systole, $\mathrm{IL}-6=$ Interleukin $6, \mathrm{IL}-8=$ Interleukin $8, \mathrm{IQR}=$ Interquartile range $\left(3^{\text {rd }}\right.$ quartile $-1^{\text {st }}$ quartile), Max. = maximum, MCP = Metacarpo-phalangeal joint, MCP-1 = macrophage chemoattractant protein 1, Min. = minimum, MIP-1b = Macrophage inflammatory protein 1 beta, no. = number , PIP = Proximal interphalangeal joint, RAMRIS BME Score = Rheumatoid Arthritis Magnetic Ressonance Bone Marrow Oedema Score.

${ }^{1}$ Mean \pm Standard deviation

synovectomised (wrist, MCP or PIP), synovectomy position (ulnar, central, radial or mixed for pooled synovectomy positions) and side (left or right).

\section{Associations between DAS-28-CRP scores and synovial explant mediator production}

Ancillary analyses using Spearman's rank correlations showed that IL-6 $(r=0.52, P=0.008)$, IL-8 $(r=0.46$,
$P=0.026)$ and MCP-1 $(r=0.44, P=0.029)$ were statistically significantly correlated to DAS-28-CRP score. The correlation of MIP-1 $\beta$ to DAS-28-CRP score did not reach statistical significance $(r=0.28, P=0.169)$.

\section{Discussion}

In this study of the hand joints of RA patients, the degree of synovitis-estimated by CDUS using the $\mathrm{CF}_{\max }$ and by 

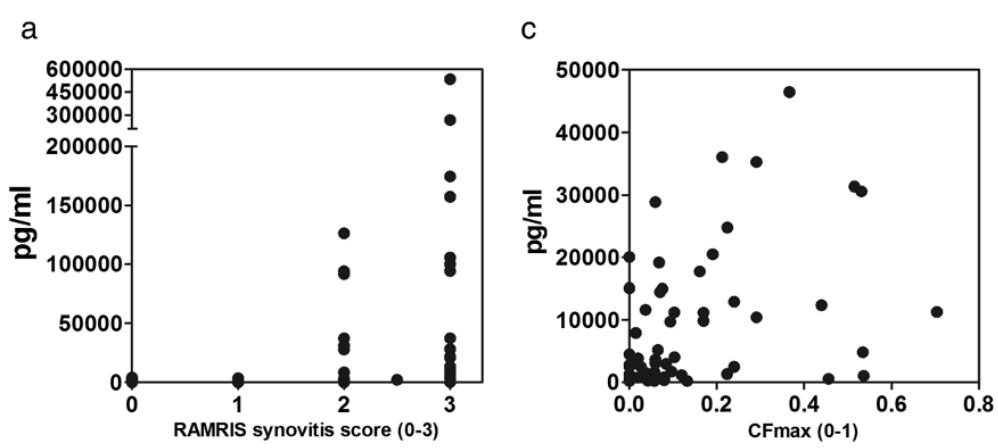

b

d
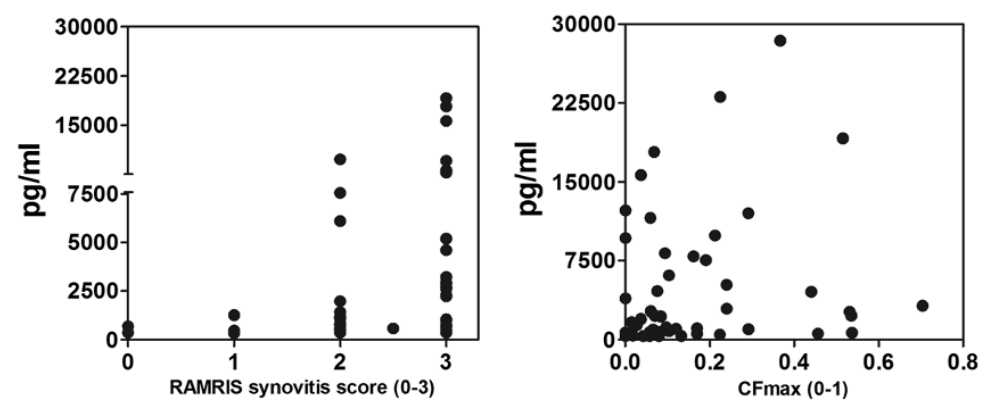

Figure 2 Scatterplots depicting synovitis, defined as synovial perfusion by maximal systolic colour fraction and by magnetic resonance imaging using the rheumatoid arthritis magnetic resonance imaging score for synovitis vs. synovial explant release after 72 hours in culture. The statistically significant plots are shown. For the remaining plots, see Additional file 3. Rheumatoid arthritis magnetic resonance imaging scores (RAMRIS) for synovitis are shown in (a) and (b). (a) Interleukin $6(P=0.04$, approximate $\rho=0.50)$. (b) Macrophage inflammatory protein $1 \beta(P=0.02$, approximate $\rho=0.63)$. Maximal systolic colour fraction $\left(C F_{\max }\right)$ values are shown in (c) and (d). (c) Monocyte

chemoattractant protein $1(P=0.009$, approximate $\rho=0.41)$. (d) Macrophage inflammatory protein $1 \beta(P=0.02$, approximate $\rho=0.38)$.

MRI using the RAMRIS criteria-was associated with synovial explant production of key inflammatory mediators. This finding supports previously published observations that DUS and MRI are sensitive imaging modalities for the detection of local inflammation. Among the four mediators investigated in our present study, we observed an interesting polarisation. Whereas IL-6, MCP-1 and
MIP-1 $\beta$ were associated with synovitis detected by MRI or $\mathrm{CF}_{\max }$ in combination, only MCP-1 and IL- 6 were associated with the extent of BME and bone erosions visualised by MRI. Furthermore IL-6, IL- 8 and MCP-1 production were correlated with DAS-28-CRP score, indicating that the local synovial mediator production reflects systemic disease.

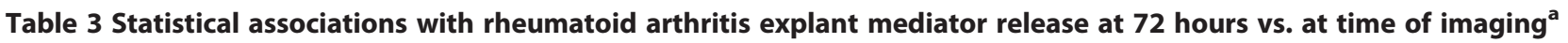

\begin{tabular}{|c|c|c|c|c|}
\hline Imaging/mediator & $\begin{array}{l}\mathrm{CF}_{\max } \\
P \text {-value (approximate } \rho \text { ) }\end{array}$ & $\begin{array}{l}\text { RAMRIS synovitis } \\
P \text {-value (approximate } \rho \text { ) }\end{array}$ & $\begin{array}{l}\text { RAMRIS BME } \\
P \text {-value (approximate } \rho \text { ) }\end{array}$ & $\begin{array}{l}\text { RAMRIS erosion } \\
P \text {-value (approximate } \rho \text { ) }\end{array}$ \\
\hline MCP-1 & $0.009(0.41)$ & $0.17(0.48)$ & $0.01(0.42)$ & $0.03(0.31)$ \\
\hline IL-6 & $0.23(0.22)$ & $0.04(0.50)$ & $0.04(0.25)$ & $0.03(0.35)$ \\
\hline IL-8 & $0.09(0.27)$ & $0.08(0.58)$ & $0.16(0.27)$ & $0.07(0.43)$ \\
\hline MIP-1 $\beta$ & $0.02(0.38)$ & $0.02(0.63)$ & $0.95(0.35)$ & $0.62(0.30)$ \\
\hline
\end{tabular}

${ }^{a} \mathrm{CF}_{\max }$, Maximal systolic colour fraction; BME, Bone marrow oedema; IL, Interleukin; MCP-1, Monocyte chemoattractant protein 1; MIP-1, Macrophage inflammatory protein $1 \beta$; RAMRIS, Rheumatoid arthritis magnetic resonance imaging score.

A mixed model has been used for the statistical analysis: $\mathrm{P}<0.05$ was considered significant. In the reduced model, covariates were excluded if $\mathrm{P}>0.10$. This table contains the data from the reduced models. The full models including stepwise covariate elimination steps and model optimization transformation can be found in the supplementary files.

The pre-specified covariates included in the statistical model: Joint Synovectomized =Wrist, MCP or PIP; Synovectomy position = Ulnar, central, radial, or mixed for pooled synovectomy positions; Side = left or right;

Approx. rho = approximated Spearman's rho; RAMRIS BME = RAMRIS bone marrow edema component; RAMRIS Erosion = RAMRIS erosion score component; RAMRIS synovitis = RAMRIS synovitis score component; IL-6 = Interleukin 6; IL-8 = Interleukin 8, MCP-1 = Monocyte chemoattractant protein 1; MIP-1b= Macrophage Inflammatory Protein 1 beta. 


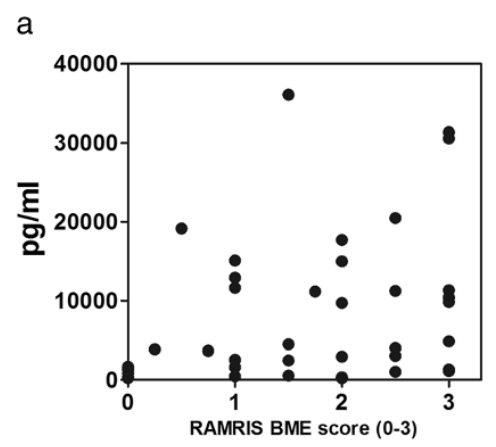

b

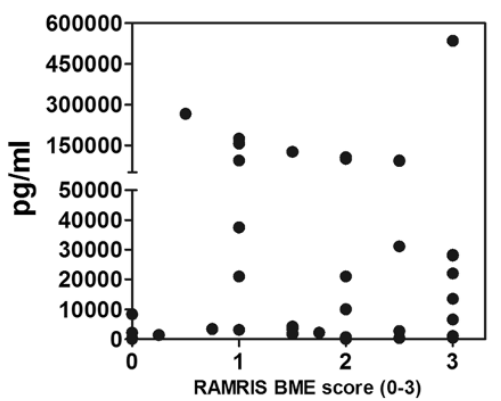

C

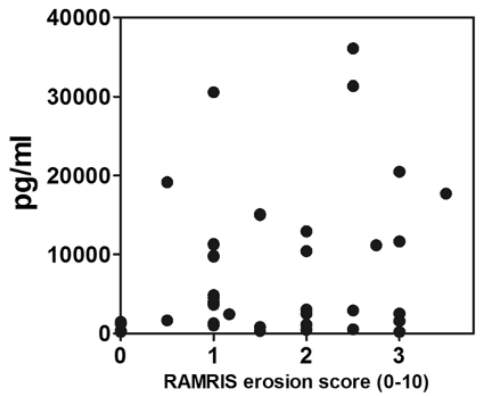

d

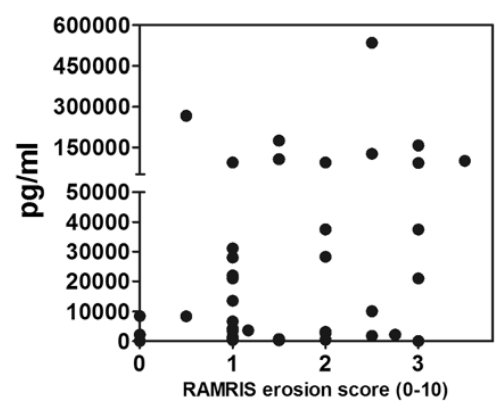

Figure 3 Scatterplots depicting bone marrow oedema and bone erosion measured by the rheumatoid arthritis magnetic resonance imaging score vs. synovial explant release after $\mathbf{7 2}$ hours in culture. The statistically significant plots are shown. For the remaining plots, see Additional file 4. Bone marrow oedema (BME) rheumatoid arthritis magnetic resonance imaging scores (RAMRIS) are shown in (a) and (b). (a) Monocyte chemoattractant protein $1(P=0.01$, approximate $\rho=0.42$ ). (B) Interleukin $6(P=0.04$, approximate $\rho=0.25)$. Bone erosion RAMRIS scores are shown in $(\mathbf{c})$ and $(\mathbf{d})$. (c) Monocyte chemoattractant protein $1(P=0.03$, approximate $\rho=0.31)$. (d) Interleukin $6(P=0.03$, approximate $\rho=0.35$ ).

This polarisation in synovial mediator expression is in accord with results produced in biopsy studies in which investigators examined differences in cellular populations and mediator and chemokine receptor expression at the cartilage-pannus junction (CPJ), which is believed to be the site driving the erosive process in the bones of RA patients. In biopsies, IL-6 expression was found exclusively at the CPJ, in contrast to IL-8-positive cells (primarily CD68+ macrophages), the vast majority of which were located at non-CPJ locations [36,37]. The picture is not completely clear, however, because CD68+ macrophages have been linked to radiographically visualised RA progression [38]. MCP-1 and its receptor, chemokine (C-C motif) receptor 2 , was located with the highest density near the synovial lining, in contrast to MIP-1 $\beta$, which had the most pronounced staining in synovial endothelium. MCP-1 - and MIP-1 $\beta$-positive cells, however, were located throughout the synovium [39]. The lack of correlation with DAS-28-CRP score and MIP-1 $\beta$ may indicate that synovial MIP- $1 \beta$ production has a role in RA pathogenesis that is more marginal than that of IL-8, which also had a strong signal (approximate $\rho=0.58$ ) to MRIdetected synovitis. In contrast to MIP- $1 \beta$, IL- 8 was statistically significantly associated with DAS-28-CRP score.
However, further studies are needed to verify the clinical significance of the cytokine expression pattern in the synovium.

The lack of association between BME and bone erosion with synovial IL- 8 and MIP-1 $\beta$ release can be explained by the main production of these mediators' originating from non-CPJ areas in the synovium. Researchers in two large clinical studies reported a lack of association of radiographic outcome and synovitis in anti-tumour necrosis factor $\alpha$ (anti-TNF- $\alpha$ )-treated cohorts $[40,41]$. These findings support our observations of different implications of these mediators in RA pathogenesis and suggest that changes in mediator profiles upon treatment may be able to predict and stratify disease outcomes with regard to synovitis and bone destruction.

Some limitations should be taken into account concerning day-to-day variations in our study. The RA patients in our study had DUS and MRI performed 24 hours prior to surgery, and day-to-day variations may have affected the reported associations [42]. Furthermore, the experimental setup did not include synovectomies from healthy controls. Therefore, baseline information on mediator release from 'normal' synovium was not possible. However, cytokine profiling of synovial fluid from healthy controls using 
a similar Bio-Plex assay setup (Bio-Rad Laboratories, Hercules, CA, USA) showed that the synovial fluid quantities of the four mediators were extremely low, with MCP-1 having the highest mean concentration at $0.5 \pm 0.4$ $\mathrm{pg} / \mathrm{ml}$ [30]. We therefore believe that cytokine levels above assay detection limits (lowest detection limit of IL-8 $=26$ $\mathrm{pg} / \mathrm{ml}$ ) should reflect synovial pathology. Taking the very low synovial fluid cytokine concentrations into account, the relatively high cutoff values for the assay's lowest detection limits may have resulted in some overestimation of synovial explant cytokine release. Considering the generally high concentration of synovial explant mediator release, an assay imprecision in the range of 26 to $360 \mathrm{pg} /$ $\mathrm{ml}$, depending on the cytokine, should not affect the overall associations with regard to imaging.

The results of this study show a noteworthy variation in synovial cytokine production and corresponding imaging pathology. The absence of signalling on DUS scans does not rule out synovial cytokine production. These findings are in accord with the results of our previous study in which DUS-detected activity was compared to synovial histopathology in a similar group of RA patients [35]. That previous study did not include grading of the synovial hypertrophy, because the images were obtained only for evaluation of colour Doppler. Further research on the association of synovial hypertrophy with synovial inflammation may provide important insights into RA disease pathogenesis.

Cellular stress inflicted by the in vitro circumstances could perhaps have skewed the mediator release from the explant cultures, resulting in higher mediator concentrations. This phenomenon did not generally occur, however, as low concentrations of synovial mediators were observed in some of the explants cultured from sites with moderate to high RAMRIS scores and in the presence of DUS-visualised activity. The observed variations may in fact have been caused by different kinetics controlling synovial perfusion, cellularity and mediator production. These factors may also account for the differences in the cytokines associated with synovial perfusion detected by $\mathrm{CF}_{\max }$ and with synovitis detected by MRI. Use of biological DMARDs (bDMARDs) may result in changes in cytokine production, but, because of the limited population size in this study, it was not possible to adjust for the different treatment modalities. Further research focused on explants and imaging in a prospective study may clarify the robustness of this method for prognostic use in bDMARD treatment. We made a great effort in designing the study to guide the area of the synovectomy on the basis of the US data; however, a completely accurate match was not possible. MRI mapping of the RAMRIS synovitis score was performed at a regional level in the wrist ( $R C$ or $M C$ ). Furthermore, BME and bone erosion data were determined as averaged values derived from the synovectomised area. This may represent a source of bias because the synovium was removed only from the dorsal part of the joint. Unfortunately, because of insufficient matching imaging data, a comparison of MRI and CDUS regarding their utility in detecting synovitis was not possible.

The reason IL-8 did not reach a statistically significant association with the MRI synovitis score is likely due to the large number of wells (52\%) above the assay's upper detection limit. None of the other cytokine levels were above the assay detection limit. Among the wells that were above detection levels, an MRI-based synovitis score of 3 was found in $72 \%$, whereas the remaining wells had a score of 2 . Budget considerations and the high dilution factors limited the possibilities of including several key inflammatory mediator candidates for measurement, despite their presence in supernatants. These candidate mediators included TNF- $\alpha$, vascular endothelial growth factor, matrix metalloprotease 3 , tissue inhibitor of metalloprotease 1 , IL-10 and interferon $\gamma$. The synovial explant model therefore offers significant information regarding synovial inflammatory activity. Interestingly, IL-1 $\beta$ levels were very low and under the detection limit $(0.57 \mathrm{pg} / \mathrm{ml})$ in five of eight patients in the initial screening. A recent publication also described low levels of IL-1 $\beta$ and TNF- $\alpha$ based on a whole-tissue synovial explant system [43]. This information is in contrast to reports of studies in which enzymedigested synovial tissue was used, possibly due to changes induced by the digestion process (for example, inhibition of formation of three-dimensional cell layers or lipopolysaccharides in the collagenase, which are potent inducers of IL-1 $\beta$ and TNF- $\alpha$ ) [44].

All patients included in our present study had synovial pathology defined by synovial hypertrophy visualised on US scans. Several sites were without DUS-visualised activity, and one case (three synovial explant positions) was judged to have no synovitis on the basis of MRI. Our findings emphasise the importance of considering the degree of greyscale synovitis when evaluating RA patients on the basis of US in the clinic.

\section{Conclusion}

In this study, we show that MRI and CDUS are highly sensitive tools for the detection of synovial pathology. Furthermore, synovial explants can identify imaging biomarkers associated with the RA disease hallmarks synovitis, BME and bone erosions, and the production of several proinflammatory cytokines correlates with overall disease activity, indicating great potential for the use of synovial explant assays in the quest for the identification of novel pathways to aid in understanding RA pathology. 


\section{Additional files}

Additional file 1: Table depicting the anatomic landmarks defining each ultrasound scan position. Standardized ultrasound scanning planes, image selection, and image quantification. Description of data: overview of the standardized ultrasound scanning planes and description of the colour Doppler quantification method.

Additional file 2: Table giving an overview of the multiplex panel chosen for the supernatant analysis and description of the multiplex panel and rationale behind the selection of synovial mediators used in the study.

Additional file 3: Figure showing scatterplots of statistically insignificant associations of synovial mediator release and $\mathrm{CF}_{\max }$ and RAMRIS synovitis score. Scatterplots depict synovitis, defined as synovial perfusion by colour fraction max ( $\mathrm{CF}_{\max }$ ) and the RAMRIS synovitis score vs. synovial explant release (in $\mathrm{pg} / \mathrm{ml}$ ) after 72 hours of culture that did not reach a statistically significant association. For $C F_{\text {max }}$ : (a) IL-6 ( $P=0.23$, approximated $\rho=0.22)$. (b) IL-8 $(P=0.09$, approximated $\rho=0.27)$. For the RAMRIS synovitis score: (c) MCP-1 $(P=0.17$, approximated $\rho=0.48)$. (d) IL-8 $(P=0.05$, approximated $\rho=0.58$ ). IL, Interleukin; MCP-1, Monocyte chemoattractant protein 1; RAMRIS, Rheumatoid arthritis magnetic resonance imaging score

Additional file 4: Figure showing scatterplots of statistically insignificant associations of synovial mediator release and RAMRIS BME and RAMRIS erosion scores. Scatterplots depict bone erosion measured by the RAMRIS bone marrow oedema (BME) and bone erosion score vs. synovial explant release $(\mathrm{pg} / \mathrm{ml})$ after 72 hours in culture that did not reach statistical significance. RAMRIS BME: (a) IL-8 $(P=0.16$, approximated $\rho=0.27)$. (b) MIP-1 $\beta(P=0.95$, approximated $\rho=0.35)$. RAMRIS erosion: (c) IL-8 $(P=0.07$, approximated $\rho=0.43)$. (d) MIP-1 $\beta(P=0.62$, approximated $\rho=0.30$ ). IL, Interleukin; MIP-1 $\beta$, Monocyte inflammatory protein $1 \beta$; RAMRIS, Rheumatoid arthritis magnetic resonance imaging score

Additional file 5: Table providing an overview of the stepwise covariate elimination in the statistical models with regard to synovial mediator production and CDUS activity. This table depicts the statistical associations between colour Doppler ultrasound ( $p=$ CDUS) activity and synovial explant mediator release after 72 hours in culture. A mixed model has been used for the statistical analysis. $P<0.05$ was considered significant. In the reduced model, covariates were excluded if the $P$-value was $>0.10$. All of the four prespecified covariates tested in the models are shown.

Additional file 6: Table giving an overview of the stepwise covariate elimination in the statistical models with regard to synovial mediator production and RAMRIS synovitis score. This table depicts the statistical associations between the rheumatoid arthritis magnetic resonance imaging synovitis score (RAMRIS) component in the part of the joint that was synovectomised and synovial explant mediator release after 72 hours in culture. A mixed model was used for the statistical analysis. $P<0.05$ was considered significant. In the reduced model, covariates were excluded when $P$-values were $>0.10$. All of the four prespecified covariates tested in the models are shown.

Additional file 7: Table providing an overview of the stepwise covariate elimination in the statistical models with regard to synovial mediator production and RAMRIS BME score. This table depicts the statistical associations between the rheumatoid arthritis magnetic resonance imaging bone marrow oedema score (focal RAMRIS BME) component and synovial explant mediator release after 72 hours in culture. A mixed model was used for the statistical analysis. $P<0.05$ was considered significant. In the reduced model, covariates were excluded if $P$-values were $>0.10$. All of the four prespecified covariates tested in the models are shown

Additional file 8: Table depicting the statistical associations between the rheumatoid arthritis magnetic resonance imaging erosion score (RAMRIS) component in the part of the joint that was synovectomised and synovial explant mediator release after $\mathbf{7 2}$ hours in culture.

Overview of the stepwise covariate elimination in the statistical models with regards to synovial mediator production and the RAMRIS erosion score. A mixed model was used for the statistical analysis. $P<0.05$ was considered significant. In the reduced model, covariates were excluded if $P>0.10$. All of the four prespecified covariates tested in the models are shown.

\section{Abbreviations}

BME: Bone marrow oedema; CD68+: Cluster of differentiation 68-positive; CDUS: Colour Doppler ultrasound; $\mathrm{CF}_{\max }$ : Maximal systolic colour fraction; CM: Complete medium; CPJ: Cartilage pannus junction; CRP: C-reactive protein; HA: Heterophilic antibody; HCQ: Hydroxychloroquine; IL: Interleukin; IQR: Interquartile range; LFU: Leflunomide; MC: Midcarpal; MCP: Metacarpal phalangeal; MCP-1: Monocyte chemoattractant protein 1; MIP-

13: Macrophage inflammatory protein 1 $\beta$; MMP-3: Matrix metalloprotease 3; MRI: Magnetic resonance imaging; MTX: Methotrexate; PIP: Proximal interphalangeal; Q1: First quartile; Q3: Third quartile; RAMRIS: Rheumatoid arthritis magnetic resonance imaging score; RC: Radiocarpal; ROI: Region of interest; SD: Standard deviation; STIR: Short tau inverted recovery; SZS: Sulphasalazine; TIMP-1: Tissue inhibitor of metalloprotease 1; TNF-a: Tumour necrosis factor a; VAS: Visual Analogue Scale; VEGF: Vascular endothelial growth factor.

\section{Competing interests}

During the course of this study Martin Andersen, Kalle Söderstöm, Pieter Spee, Ulrik GW Mørch, and Lars Karlsson were employed at Novo Nordisk. Kalle Söderstöm, Pieter Spee, Ulrik GW Mørch, and Lars Karlsson owned stocks in Novo Nordisk. MA, KS, UGWM, PS and LK were all employed by Novo Nordisk during the patient recruitment period. KS, PS, UGWM and LK owned stocks in Novo Nordisk.

\section{Authors' contributors}

MA was responsible for the study conception and design, manuscript writing, establishment of the synovial explant assay, data collection and analysis and critical revision of the manuscript. MB was responsible for the study conception and design, manuscript writing, data collection and analysis and critical revision of the manuscript. KE and STP were responsible for the study conception and design, data collection and analysis and critical revision of the manuscript. RC was responsible for the study conception and design, manuscript writing, statistical analyses and critical revision of the manuscript. KS, EMB, NV was responsible for the study conception and design and critical revision of the manuscript. NS was responsible for the study conception and design, manuscript writing, data collection and critical revision of the manuscript. PS, UM, BDS, LK and HB were responsible for the study conception and design, securing funding and critical revision of the manuscript. All authors read and approved the final manuscript.

\section{Acknowledgments}

The authors would like to thank the study participants as well as Inger Wätjen, Eva Littrup Andersen, Mette Okkels, Jette Møller Frøsig and Suzi Høeg Madsen. The study was supported by unrestricted grants from Novo Nordisk; The Danish Agency for Science, Technology and Innovation; The Oak Foundation; and all the centres involved in the study, which were The Parker Institute; the Department of Rheumatology, Copenhagen University Hospital, Bispebjerg and Frederiksberg, Copenhagen; Translational Immunology, Biopharmaceutical Research Unit, Måløv, Denmark; Novo Nordisk, Copenhagen; the Department of Radiology, Copenhagen University Hospital, Bispebjerg and Frederiksberg, Copenhagen; the Section of Hand Surgery, Department of Orthopedics, Gentofte University Hospital, Hellerup, Denmark; Biomarkers, Novo Nordisk, Søborg, Denmark; and the Department of Anesthesiology, Intensive Care and Operations, Gentofte University Hospital. The Parker Institute is supported by grants from the Oak Foundation, a nonprofit funding source that had no role in the design or conduct of the study; the collection, analysis and interpretation of the data; or the preparation, review and approval of the version of the manuscript submitted for publication.

\section{Author details}

${ }^{1}$ The Parker Institute, Department of Rheumatology, Copenhagen University Hospital, Bispebjerg and Frederiksberg, Nordre Fasanvej 57, Copenhagen 2000 Frederiksberg, Denmark. ${ }^{2}$ Department of Translational Immunology, Biopharmaceutical Research Unit, Novo Nordisk, Novo Nordisk Park 1, 2760 Måløv, Denmark. ${ }^{3}$ Department of Radiology, Copenhagen University Hospital, Bispebjerg and Frederiksberg, Bispebjerg Bakke 23, Copenhagen 2400, København NV, Denmark. ${ }^{4}$ Department of Orthopedics, Section of Hand Surgery, Gentofte University Hospital, Gentofte Hospital, Niels Andersens Vej 65, 2900 Hellerup, Denmark. ${ }^{5}$ Biomarkers, Novo Nordisk, Vandtårnsvej 108, 2860 Søborg, Denmark. ${ }^{6}$ Department of Anaesthesiology, Intensive Care and 
Operations, Gentofte University Hospital, Gentofte Hospital, Niels Andersens Vej 65, 2900 Hellerup, Denmark.

Received: 23 November 2013 Accepted: 4 April 2014

Published: 5 May 2014

\section{References}

1. Schipper LG, Vermeer M, Kuper HH, Hoekstra MO, Haagsma CJ, Den Broeder AA, van Riel P, Fransen J, van de Laar MAFJ: A tight control treatment strategy aiming for remission in early rheumatoid arthritis is more effective than usual care treatment in daily clinical practice: a study of two cohorts in the Dutch Rheumatoid Arthritis Monitoring registry. Ann Rheum Dis 2012, 71:845-850.

2. Smolen JS, Landewé R, Breedveld FC, Dougados M, Emery P, Gaujoux-Viala C, Gorter S, Knevel R, Nam J, Schoels M, Aletaha D, Buch M, Gossec L, Huizinga T, Bijlsma JW, Burmester G, Combe B, Cutolo M, Gabay C, Gomez-Reino J, Kouloumas M, Kvien TK, Martin-Mola E, Mclnnes I, Pavelka K, van Riel P, Scholte M, Scott DL, Sokka T, Valesini G, et al: EULAR recommendations for the management of rheumatoid arthritis with synthetic and biological disease-modifying antirheumatic drugs. Ann Rheum Dis 2010, 69:964-975. A published erratum appears in Ann Rheum Dis 2011, 70:1519.

3. Brown AK, Quinn MA, Karim Z, Conaghan PG, Peterfy CG, Hensor E, Wakefield RJ, O'Connor PJ, Emery P: Presence of significant synovitis in rheumatoid arthritis patients with disease-modifying antirheumatic drug-induced clinical remission: evidence from an imaging study may explain structural progression. Arthritis Rheum 2006, 54:3761-3773.

4. Brown AK, Conaghan PG, Karim Z, Quinn MA, Ikeda K, Peterfy CG, Hensor E, Wakefield RJ, O'Connor PJ, Emery P: An explanation for the apparent dissociation between clinical remission and continued structural deterioration in rheumatoid arthritis. Arthritis Rheum 2008, 58:2958-2967.

5. Aletaha D, Neogi T, Silman AJ, Funovits J, Felson DT, Bingham CO 3rd, Birnbaum NS, Burmester GR, Bykerk VP, Cohen MD, Combe B, Costenbader KH, Dougados M, Emery P, Ferraccioli G, Hazes JM, Hobbs K, Huizinga TW, Kavanaugh A, Kay J, Kvien TK, Laing T, Mease P, Ménard HA, Moreland LW, Naden RL, Pincus T, Smolen JS, Stanislawska-Biernat E, Symmons D, et al: Rheumatoid arthritis classification criteria: an American College of Rheumatology/European League Against Rheumatism collaborative initiative. Arthritis Rheum 2010, 62:2569-2581.

6. Haavardsholm EA, Bøyesen P, Østergaard M, Schildvold A, Kvien TK: Magnetic resonance imaging findings in 84 patients with early rheumatoid arthritis: bone marrow oedema predicts erosive progression. Ann Rheum Dis 2008, 67:794-800.

7. Østergaard M, Edmonds J, McQueen F, Peterfy C, Lassere M, Ejbjerg B, Bird P, Emery P, Genant $H$, Conaghan P: An introduction to the EULAR-OMERACT rheumatoid arthritis MRI reference image atlas. Ann Rheum Dis 2005, 64:i3-i7.

8. Peterfy C, Østergaard M, Conaghan PG: MRI comes of age in RA clinical trials. Ann Rheum Dis 2013, 72:794-796.

9. Haringman JJ, Gerlag DM, Zwinderman AH, Smeets TJ, Kraan MC, Baeten D, Mclnnes IB, Bresnihan B, Tak PP: Synovial tissue macrophages: a sensitive biomarker for response to treatment in patients with rheumatoid arthritis. Ann Rheum Dis 2005, 64:834-838.

10. Gaffney K, Cookson J, Blake D, Coumbe A, Blades S: Quantification of rheumatoid synovitis by magnetic resonance imaging. Arthritis Rheum 1995, 38:1610-1617

11. Østergaard M, Stoltenberg M, Løvgreen-Nielsen P, Volck B, Jensen CH, Lorenzen I: Magnetic resonance imaging-determined synovial membrane and joint effusion volumes in rheumatoid arthritis and osteoarthritis: comparison with the macroscopic and microscopic appearance of the synovium. Arthritis Rheum 1997, 40:1856-1867.

12. Axelsen MB, Stoltenberg M, Poggenborg RP, Kubassova O, Boesen M, Bliddal H, Hørslev-Petersen K, Hanson LG, Østergaard M: Dynamic gadolinium-enhanced magnetic resonance imaging allows accurate assessment of the synovial inflammatory activity in rheumatoid arthritis knee joints: a comparison with synovial histology. Scand J Rheumatol 2012, 41:89-94.

13. Bugatti S, Manzo A, Caporali R, Montecucco C: Inflammatory lesions in the bone marrow of rheumatoid arthritis patients: a morphological perspective. Arthritis Res Ther 2012, 14:229.
14. Dalbeth N, Smith T, Gray S, Doyle A, Antill P, Lobo M, Robinson E, King A, Cornish J, Shalley G, Gao A, McQueen FM: Cellular characterisation of magnetic resonance imaging bone oedema in rheumatoid arthritis; implications for pathogenesis of erosive disease. Ann Rheum Dis 2009, 68:279-282

15. Jimenez-Boj E, Nöbauer-Huhmann I, Hanslik-Schnabel B, Dorotka R, Wanivenhaus AH, Kainberger F, Trattnig S, Axmann R, Tsuji W, Hermann S, Smolen J, Schett G: Bone erosions and bone marrow edema as defined by magnetic resonance imaging reflect true bone marrow inflammation in rheumatoid arthritis. Arthritis Rheum 2007, 56:1118-1124.

16. Chabaud M, Miossec $P$ : The combination of tumor necrosis factor a blockade with interleukin-1 and interleukin-17 blockade is more effective for controlling synovial inflammation and bone resorption in an ex vivo model. Arthritis Rheum 2001, 44:1293-1303.

17. Chevrel G, Garnero P, Miossec P: Addition of interleukin 1 (IL1) and IL17 soluble receptors to a tumour necrosis factor a soluble receptor more effectively reduces the production of IL6 and macrophage inhibitory protein-3a and increases that of collagen in an in vitro model of rheumatoid synoviocyte activation. Ann Rheum Dis 2002, 61:730-733.

18. Hosaka K, Ryu J, Saitoh S, Ishii T, Kuroda K, Shimizu K: The combined effects of anti-TNFa antibody and IL-1 receptor antagonist in human rheumatoid arthritis synovial membrane. Cytokine 2005, 32:263-269.

19. Miossec P, Briolay J, Dechanet J, Wijdenes J, Martinez-Valdez H, Banchereau $\mathrm{J}$ : Inhibition of the production of proinflammatory cytokines and immunoglobulins by interleukin-4 in an ex vivo model of rheumatoid synovitis. Arthritis Rheum 1992, 35:874-883.

20. Miossec $P$, Chomarat $P$, Dechanet J, Moreau JF, Roux JP, Delmas $P$ Banchereau J: Interleukin-4 inhibits bone resorption through an effect on osteoclasts and proinflammatory cytokines in an ex vivo model of bone resorption in rheumatoid arthritis. Arthritis Rheum 1994, 37:1715-1722.

21. Schultz O, Keyszer G, Zacher J, Sittinger M, Burmester GR: Development of in vitro model systems for destructive joint diseases: novel strategies for establishing inflammatory pannus. Arthritis Rheum 1997, 40:1420-1428.

22. Ounissi-Benkalha H, Pelletier JP, Tardif G, Mineau F, Jolicoeur FC, Ranger $P$ Martel-Pelletier J: In vitro effects of 2 antirheumatic drugs on the synthesis and expression of proinflammatory cytokines in synovial membranes from patients with rheumatoid arthritis. J Rheumatol 1996, 23:16-23.

23. Volin MV, Campbell PL, Connors MA, Woodruff DC, Koch AE: The effect of sulfasalazine on rheumatoid arthritic synovial tissue chemokine production. Exp Mol Pathol 2002, 73:84-92.

24. Nic an Ultaigh S, Saber TP, McCormick J, Connolly M, Dellacasagrande J, Keogh B, McCormack W, Reilly M, O'Neill LA, McGuirk P, Fearon U, Veale DJ: Blockade of Toll-like receptor 2 prevents spontaneous cytokine release from rheumatoid arthritis ex vivo synovial explant cultures. Arthritis Res Ther 2011, 13:R33.

25. van de Sande MG, Gerlag DM, Lodde BM, van Baarsen LG, Alivernini S, Codullo V, Felea I, Vieira-Sousa E, Fearon U, Reece R, Montecucco C, Veale DJ, Pitzalis C, Emery P, Klareskog L, Mclnnes IB, Tak PP: Evaluating antirheumatic treatments using synovial biopsy: a recommendation for standardisation to be used in clinical trials. Ann Rheum Dis 2011, 70:423-427

26. Scirè CA, Epis O, Codullo V, Humby F, Morbini P, Manzo A, Caporali R, Pitzalis C, Montecucco C: Immunohistological assessment of the synovial tissue in small joints in rheumatoid arthritis: validation of a minimally invasive ultrasound-guided synovial biopsy procedure. Arthritis Res Ther 2007, 9:R101.

27. Todd DJ, Knowlton N, Amato M, Frank MB, Schur PH, Izmailova ES, Roubenoff R, Shadick NA, Weinblatt ME, Centola M, Lee DM: Erroneous augmentation of multiplex assay measurements in patients with rheumatoid arthritis due to heterophilic binding by serum rheumatoid factor. Arthritis Rheum 2011, 63:894-903.

28. Qvistgaard E, Røgind H, Torp-Pedersen S, Terslev L, Danneskiold-Samsøe B, Bliddal $\mathrm{H}$ : Quantitative ultrasonography in rheumatoid arthritis: evaluation of inflammation by Doppler technique. Ann Rheum Dis 2001, 60:690-693.

29. Arnett FC, Edworthy SM, Bloch DA, McShane DJ, Fries JF, Cooper NS, Healey LA, Kaplan SR, Liang MH, Luthra HS, Medsger TA, Jr Mitchell DM, Neustadt DH, Pinals RS, Schaller JG, Sharp JT, Wilder RL, Hunder GG: The American Rheumatism Association 1987 revised criteria for the classification of rheumatoid arthritis. Arthritis Rheum 1988, 31:315-324 
30. Cuellar VG, Cuellar JM, Golish SR, Yeomans DC, Scuderi GJ: Cytokine profiling in acute anterior cruciate ligament injury. Arthroscopy 2010, 26:1296-1301.

31. Terslev L, von der Recke P, Torp-Pedersen S, Koenig MJ, Bliddal H: Diagnostic sensitivity and specificity of Doppler ultrasound in rheumatoid arthritis. J Rheumatol 2008, 35:49-53.

32. Torp-Pedersen ST, Terslev L: Settings and artefacts relevant in colour/ power Doppler ultrasound in rheumatology. Ann Rheum Dis 2008 , 67:143-149.

33. Ellegaard KM, Torp-Pedersen S, Christensen R, Stoltenberg M, Hansen A, Lorenzen T, Jensen DV, Lindegaard H, Juul L, Røgind H, Bülow P, Chrysidis S, Kowalski M, Danneskiold-Samsøe B, Bliddal H: Feasibility of a standardized ultrasound examination in patients with rheumatoid arthritis: a quality improvement among rheumatologists cohort. BMC Musculoskelet Disord 2012, 13:35.

34. Laurell L, Court-Payen, Nielsen S, Zak M, Boesen M, Fasth A: Comparison of ultrasonography with Doppler and MRI for assessment of disease activity in juvenile idiopathic arthritis: a pilot study. Pediatr Rheumatol Online J 2012, 10:23.

35. Andersen M, Ellegaard K, Hebsgaard JB, Christensen R, Torp-Pedersen S, Kvist PH, Søe N, Rømer J, Vendel N, Bartels EM, Danneskiold-Samsøe B, Bliddal H: Ultrasound colour Doppler is associated with synovial pathology in biopsies from hand joints in rheumatoid arthritis patients: a cross-sectional study. Ann Rheum Dis 2014, 73:678-683.

36. Deleuran B, Lemche P, Kristensen M, Chu CQ, Field M, Jensen J, Matsushima K, Stengaard-Pedersen K: Localisation of interleukin 8 in the synovial membrane, cartilage-pannus junction and chondrocytes in rheumatoid arthritis. Scand J Rheumatol 1994, 23:2-7.

37. Chu CQ, Field M, Allard S, Abney E, Feldmann M, Maini RN: Detection of cytokines at the cartilage/pannus junction in patients with rheumatoid arthritis: implications for the role of cytokines in cartilage destruction and repair. Br J Rheumatol 1992, 31:653-661.

38. Mulherin D, FitzGerald O, Bresnihan B: Synovial tissue macrophage populations and articular damage in rheumatoid arthritis. Arthritis Rheum 1996, 39:115-124.

39. Katschke KJ Jr, Rottman JB, Ruth JH, Qin S, Wu L, LaRosa G, Ponath P, Park CC, Pope RM, Koch AE: Differential expression of chemokine receptors on peripheral blood, synovial fluid, and synovial tissue monocytes/ macrophages in rheumatoid arthritis. Arthritis Rheum 2001, 44:1022-1032.

40. Smolen JS, Han C, Bala M, Maini RN, Kalden JR, van der Heijde D, Breedveld FC, Furst DE, Lipsky PE, ATTRACT Study Group: Evidence of radiographic benefit of treatment with infliximab plus methotrexate in rheumatoid arthritis patients who had no clinical improvement: a detailed subanalysis of data from the anti-tumor necrosis factor trial in rheumatoid arthritis with concomitant therapy study. Arthritis Rheum 2005, 52:1020-1030.

41. Landewé R, van der Heijde D, Klareskog L, van Vollenhoven R, Fatenejad S: Disconnect between inflammation and joint destruction after treatment with etanercept plus methotrexate: results from the trial of etanercept and methotrexate with radiographic and patient outcomes. Arthritis Rheum 2006, 54:3119-3125.

42. Semerano L, Gutierrez M, Falgarone G, Filippucci E, Guillot X, Boissier MC Grassi W: Diurnal variation of power Doppler in metacarpophalangeal joints of patients with rheumatoid arthritis: a preliminary study. Ann Rheum Dis 2011, 70:1699-1700.

43. Kontny E, Plebanczyk M, Lisowska B, Olszewska M, Maldyk P, Maslinski W: Comparison of rheumatoid articular adipose and synovial tissue reactivity to proinflammatory stimuli: contribution to adipocytokine network. Ann Rheum Dis 2012, 71:262-267.

44. Nozaki T, Takahashi K, Ishii O, Endo S, Hioki K, Mori T, Kikukawa T, Boumpas DT, Ozaki S, Yamada H: Development of an ex vivo cellular model of rheumatoid arthritis: critical role of CD14-positive monocyte/macrophages in the development of pannus tissue. Arthritis Rheum 2007, 56:2875-2885.

\section{doi:10.1186/ar4557}

Cite this article as: Andersen et al:: Synovial explant inflammatory mediator production corresponds to rheumatoid arthritis imaging hallmarks: a cross-sectional study. Arthritis Research \& Therapy 2014 16:R107.

\section{Submit your next manuscript to BioMed Central and take full advantage of:}

- Convenient online submission

- Thorough peer review

- No space constraints or color figure charges

- Immediate publication on acceptance

- Inclusion in PubMed, CAS, Scopus and Google Scholar

- Research which is freely available for redistribution 